\title{
Dimensional Reduction of Conformal Tensors and Einstein-Weyl Spaces ${ }^{\star}$
}

\author{
Roman JACKIW
}

Department of Physics, Massachusetts Institute of Technology, Cambridge, MA 02139, USA

E-mail: jackiw@mit.edu

Received August 29, 2007, in final form September 16, 2007; Published online September 21, 2007

Original article is available at http://www.emis.de/journals/SIGMA/2007/091/

\begin{abstract}
Conformal Weyl and Cotton tensors are dimensionally reduced by a KaluzaKlein procedure. Explicit formulas are given for reducing from four and three dimensions to three and two dimensions, respectively. When the higher dimensional conformal tensor vanishes because the space is conformallly flat, the lower-dimensional Kaluza-Klein functions satisfy equations that coincide with the Einstein-Weyl equations in three dimensions and kink equations in two dimensions.
\end{abstract}

Key words: conformal tensors; dimensional reductions; Kaluza-Klein

2000 Mathematics Subject Classification: $81 \mathrm{~T} 40$

\section{Introduction}

In this paper, we shall examine a mathematical problem, which does not possess any evident physical significance, but nevertheless leads to interesting equations that lie in various areas of mathematical physics. The problem that we address concerns the dimensional reduction of geometric conformal tensors (defined below) from $n$ to $n-1$ dimensions. More specifically, for an $n$-dimensional conformal tensor, constructed from a metric tensor $g_{M N},\{M, N\}: 0,1, \ldots, n-1$, the metric tensor is parameterized in the Kaluza-Klein fashion

$$
g_{M N}=e^{2 \sigma}\left(\begin{array}{rr}
g_{\mu \nu}-a_{\mu} a_{\nu} & -a_{\mu} \\
-a_{\nu} & -1
\end{array}\right), \quad\{\mu, \nu\}: 0,1, \ldots, n-2,
$$

corresponding to the $n$ and $n-1$ line elements

$$
\begin{aligned}
& d s_{(n)}^{2}=g_{M N} d x^{M} d x^{N}=e^{2 \sigma}\left[d s_{n-1}^{2}-\left(a_{\mu} d x^{\mu}+d x^{n-1}\right)^{2}\right], \\
& d s_{(n-1)}^{2}=g_{\mu \nu} d x^{\mu} d x^{\nu} .
\end{aligned}
$$

The metric functions are taken to be independent of the "last" coordinate $x^{n-1}$. Consequently the $n$-dimensional conformal tensor reduces to an $n-1$ dimensional conformal tensor, plus other geometrical entities appropriate to $n-1$ dimensions. An $n$-dimensional diffeomorphism, $\delta x^{N}=-f^{N}(x)$, where the transformation function is independent of the last coordinate, acts on the quantities in (1) in such a way that $\left\{g_{\mu \nu}, a_{\mu}, \sigma\right\}$ transform as $n-1$ dimensional tensors, vector and scalar, respectively, and also $a_{\mu}$ experiences an Abelian gauge transformation.

The effect of such a dimensional reduction on the $n$-dimensional Riemann tensor is known: it is described by the classic geometric Gauss-Codazzi equations (see for example [1]). Here we present analogous results for conformal tensors, in various dimensions.

We begin by describing conformal tensors.

${ }^{\star}$ This paper is a contribution to the Proceedings of the Seventh International Conference "Symmetry in Nonlinear Mathematical Physics" (June 24-30, 2007, Kyiv, Ukraine). The full collection is available at http://www.emis.de/journals/SIGMA/symmetry2007.html 


\section{Conformal tensors}

In dimensions greater than three, the conformal tensor is the Weyl tensor $C^{K L M N}$ related to the Riemann tensor by

$$
\begin{aligned}
& C^{K L M N} \equiv R^{K L M N}-\frac{1}{n-2}\left(g^{K N} S^{M L}-g^{K N} S^{M L}-g^{L M} S^{N K}+g^{L N} S^{M K}\right), \\
& S^{N L} \equiv R^{N L}-\frac{1}{2(n-1)} g^{N L} R .
\end{aligned}
$$

$R^{N L}$ and $R$ are the Ricci quantities, while $C^{K L M N}$ has the following properties

(a) It is covariant against conformal redefinition of the metric

$$
g_{M N}(x) \rightarrow \lambda(x) g_{M N}(x) .
$$

(b) It vanishes if and only if the space is conformally flat: $g_{M N}$ is diffeomorphic to $\lambda \eta_{M N}$ where $\eta_{M N}$ is flat.

(c) It possesses the symmetries of the Riemann tensor and also is traceless in each index pair.

Evidently, according to properties (a) and (b), the Weyl tensor acts as a template for conformal flatness: by evaluating it on a specific metric tensor one can learn whether the space-time is conformally flat.

In three dimensions the Weyl tensor vanishes identically, and the Riemann tensor is given by the last term in (2) (at $n=3$ ). But not all 3-dimensional space-times are conformally flat. So there is needed a replacement for the Weyl tensor, which would act as a template for conformal flatness.

The Cotton tensor

$$
C^{K L}=\frac{1}{2 \sqrt{g}}\left(\varepsilon^{K M N} D_{M} R_{N}^{L}+\varepsilon^{L M N} D_{M} R_{N}^{K}\right)
$$

serves that role, as it possesses conformal template properties (a) and (b). $C^{K L}$ is symmetric in its indices, and like the Weyl tensor, it is traceless. The Weyl tensor is not obtained by varying a scalar action. But the Cotton tensor enjoys this property, since it is the variation of the gravitational Chern-Simons term

$$
\begin{aligned}
& C S(\Gamma) \equiv \frac{1}{4 \pi^{2}} \int d^{3} x \varepsilon^{K L M}\left(\frac{1}{2} \Gamma_{K S}^{R} \partial_{L} \Gamma_{M R}^{S}+\frac{1}{3} \Gamma_{K S}^{R} \Gamma_{L T}^{S} \Gamma_{M K}^{T}\right), \\
& \delta C S(\Gamma)=-\frac{1}{4 \pi^{2}} \int d^{3} x \sqrt{g} C^{K L} \delta g_{K L} .
\end{aligned}
$$

Because $C S(\Gamma)$ is a scalar, $C^{K L}$ is covariantly conserved.

Finally in two dimensions, all spaces are (locally) conformally flat, so there is no need for a conformal tensor, and indeed none exists.

\section{Dimensional reductions}

We have derived the relevant formulas for the general $n \rightarrow n-1$ reduction. They are complicated and will not be recorded here, since they appear in the published research paper [2]. We remark on two general features

(a) The reductions of the Weyl and Cotton tensors do not depend on the conformal factor $e^{2 \sigma}$ in (1), because the tensors are conformally invariant. 
(b) The dependence of the tensors on the vector $a_{\mu}$ is mostly through the "gauge invariant" combination.

$$
f_{\mu \nu}=\partial_{\mu} a_{\nu}-\partial_{\nu} a_{\mu}
$$

We present explicit formulas for the $4 \rightarrow 3$ and $3 \rightarrow 2$ reductions. These are especially interesting owing to the absence of a Weyl tensor in three and two dimensions, and the presence of the Cotton tensor in three dimensions.

\section{$3.1 \quad 4 \rightarrow 3$ reduction}

The 4-dimensional Weyl tensor $C^{M N K L}$ with indices in the 3-dimensional range $(\mu, \nu, \lambda, \tau)$ reads

$$
C^{\mu \nu \lambda \tau}=g^{\mu \lambda} c^{\tau \nu}-g^{\mu \tau} c^{\lambda \nu}-g^{\nu \lambda} c^{\tau \mu}+g^{\nu \tau} c^{\lambda \mu}
$$

where

$$
c^{\mu \nu} \equiv \frac{1}{2}\left(r^{\mu \nu}-\frac{1}{3} g^{\mu \nu} r-f^{\mu} f^{\nu}+\frac{1}{3} g^{\mu \nu} f^{2}\right) .
$$

Here $r^{\mu \nu}$ and $r$ are 3-dimensional Ricci entities, constructed from the 3-dimensional metric tensor $g_{\mu \nu}$, while $f^{\mu}$ is the dual "field" strength, constructed from the vector potential $a_{\mu}$ $($ see $(3))$

$$
f^{\mu} \equiv \frac{\epsilon^{\mu \alpha \beta}}{2 \sqrt{g}} f_{\alpha \beta}=\frac{\varepsilon^{\mu \alpha \beta}}{\sqrt{g}} \partial_{\alpha} a_{\beta} .
$$

There remains one more component of the 4-dimensional Weyl tensor that must be specified

$$
C^{-\lambda \mu \nu}=\frac{\epsilon^{\mu \nu \tau}}{2 \sqrt{g}}\left(d^{\lambda} f_{\tau}+d_{\tau} f^{\lambda}\right)-a_{\tau} C^{\tau \lambda \mu \nu} .
$$

Here $d^{\lambda}$ is the 3-dimensional covariant derivative, and "-" refers to the "last" $(M=3)$ valued index of the 4-dimensional Weyl tensor. Other components of $C^{M N K L}$ are fixed by its tracelessness [2].

\section{$3.2 \quad 3 \rightarrow 2$ reduction}

The 3-dimensional Cotton tensor $C^{M N}$, with its indices in the 2 dimensional range, becomes

$$
C_{\mu \nu}=g_{\mu \nu}\left(d^{2} f-f^{3}-\frac{1}{2} r f\right)-d_{\mu} d_{\nu} f .
$$

Now $r$ is the 2-dimensional Ricci scalar constructed from the 2-dimensional metric tensor $g_{\mu \nu}$; $d_{\mu}$ is the appropriate 2-dimensional covariant derivative; $f$ is the dual to $f_{\mu \nu}$ - a scalar in two dimensions

$$
f \equiv \frac{\varepsilon^{\mu \nu}}{2 \sqrt{-g}} f_{\mu \nu}=\frac{\varepsilon^{\mu \nu}}{\sqrt{-g}} \partial_{\mu} a_{\nu} .
$$

The further component of the Cotton tensor is given by

$$
C^{-\mu}=\frac{1}{2 \sqrt{-g}} \varepsilon^{\mu \nu} \partial_{\nu}\left(r+3 f^{2}\right)-a_{\nu} C^{\mu \nu}
$$

with "-" denoting the "last" $(M=2)$ valued index. The remaining component of $C^{M N}$ is fixed by its tracelessness [3]. 


\section{Embedding in a conformally flat space}

If we demand that the higher dimensional conformal tensor vanishes, the reduced formulas become equations that determine the lower-dimensional metric tensor and vector field (actually only its "gauge"-invariant curl enters). The lower dimensional geometry is therefore embedded in a conformally flat space of one higher dimension.

\section{$4.1 \quad 4 \rightarrow 3$ embedding}

When the 4-dimensional Weyl tensor vanishes (4) and (5) imply the 3-dimensional traceless equation

$$
C^{\mu \nu}=0 \Rightarrow r^{\mu \nu}-\frac{1}{3} g^{\mu \nu} r=f^{\mu} f^{\nu}-\frac{1}{3} g^{\mu \nu} f^{\alpha} f_{\alpha} .
$$

While (4) and (6) require

$$
d_{\mu} f_{\nu}+d_{\nu} f_{\mu}=0
$$

i.e. $f^{\mu}$ is a Killing vector of the 3-geometry. Equations (8) and (9) have the consequence (by differention of (8) and use of (9))

$$
r=-5 f^{2}+c,
$$

where $c$ is a constant. Also one readily shows that the quantity

$$
F^{\mu} \equiv \frac{\varepsilon^{\mu \nu \lambda}}{\sqrt{g}} d_{\nu} f_{\lambda}
$$

is an additional Killing vector of the 3-geometry - we call it the "dual" Killing vector [2].

We present explicit solutions to (8) and (9) that are static and circularly symmetric. With such an Ansatz, two solutions are found [2]

$$
\text { (a) } \begin{aligned}
d s_{3}^{2} & =v(\rho) d t^{2}-\frac{4 / a}{1-\rho^{2} / a} \frac{d \rho^{2}}{v(\rho)}-\rho^{2} d \theta^{2}, \\
v(\rho) & \equiv A+B \sqrt{1-\rho^{2} / a} .
\end{aligned}
$$

$f^{\mu}$ is the time-like Killing vector for (10)

$$
f^{\mu}:\left\{f^{t}=1, f^{\rho}=0, f^{\theta}=0\right\},
$$

while the dual Killing vector is spacelike

$$
F^{\mu}:\left\{F^{t}=0, F^{\rho}=0, F^{\theta}=1\right\} .
$$

Note $|a|$ may be eliminated from (10) by rescaling $\rho$ and $\theta$.

$$
\begin{aligned}
\text { (b) } \quad d s_{3}^{2} & =w(\rho) d t^{2}-\frac{1}{w(\rho)} d \rho^{2}-\rho^{2}(d \theta)^{2}, \\
w(\rho) & \equiv \frac{1}{4} \rho^{4}+A \rho^{2}+B .
\end{aligned}
$$

Now $f^{\mu}$ is the space-like Killing vector,

$$
f^{\mu}=\left\{f^{t}=0, f^{\rho}=0, f^{\theta}=1\right\}
$$

while dual Killing vector is time-like

$$
F^{\mu}:\left\{F^{t}=1, F^{\rho}=0, F^{\theta}=0\right\} .
$$




\section{$4.2 \quad 3 \rightarrow 2$ embedding}

The vanishing of the 3-dimensional Cotton tensor requires, according to (6), both the 2-dimensional traceless equation

$$
\left(d_{\mu} d_{\nu}-\frac{g_{\mu \nu}}{2} d^{2}\right) f=0
$$

and the trace condition

$$
d^{2} f-2 f^{3}-r f=0
$$

while (7) sets

$$
r=-3 f^{2}+c
$$

where $c$ is a constant. Therefore (18) becomes [3]

$$
d^{2} f+f^{3}-c f=0
$$

Since, unlike the Weyl tensor, the Cotton tensor is the variation of an action - the gravitational Chern-Simons action $C S(\Gamma)$ - equations (17)-(20) arise by varying the dimensionally reduced $C S(\Gamma)$, which reads

$$
C S=-\frac{1}{8 \pi^{2}} \int d^{2} x \sqrt{-g}\left(f r+f^{3}\right)
$$

The equations (17)-(20) can be solved for arbitrary values of $c$ : positive, negative, zero [3, 4]. Especially interesting are the solutions for $c>0$, where the $f \leftrightarrow-f$ reflection "symmetry" of the equations (not of the action (21)) is spontaneously broken by the solution $f= \pm \sqrt{c}, r=2 c$. In further analogy with familiar field theoretical behavior, there also exists a solution which interpolates between the $\pm \sqrt{c}$ "vacua"

$$
f=\sqrt{c} \tanh \frac{\sqrt{c} x}{2}, \quad r=2 c+\frac{3 c}{\cosh ^{2} \frac{\sqrt{c}}{2} x} .
$$

It is amusing to recall the above mentioned field theoretic analog. In 2-dimensional Minkowski space-time a scalar field $\Phi$ can satisfy the equation

$$
\square \Phi+\Phi^{3}-c \Phi=0, \quad c>0
$$

which possesses the $\Phi \leftrightarrow-\Phi$ symmetry breaking solution $\Phi= \pm \sqrt{c}$. As is well known the equation also admits the kink solution which interpolates between the two $\pm \sqrt{c}$ "vacua"

$$
\psi_{\mathrm{kink}}=\sqrt{c} \tanh \sqrt{\frac{c}{2}} x
$$

The analogy is perfect, but there is a normalization discrepancy. The curved-space kink possess a spatial coordinate scaled by 2 , while in the flat-space kink the scaling is $\sqrt{2}$. This is the only effect of the non-trivial geometry. 


\section{Other work}

In $[4,5]$ we list papers that rely to some extent on the results presented here, specifically employing formulas arising in the $3 \rightarrow 2$ reduction.

In the discussion following the lecture, M. Eastwood observed that the formulas relevant to the $4 \rightarrow 3$ reduction coincide with equations that arise in the 3 -dimensional Einstein-Weyl conformal theory [6]. This is an interesting connection, which we now elaborate.

Einstein-Weyl theory (in any dimension) is equipped with a metric tensor $g_{\mu \nu}$ and an additional vector $W_{\omega}$ - the "Weyl potential" - which arises when the covariant "Weyl derivative" $\triangle_{\omega}^{W}$, involving the torsion-less "Weyl connection" $W_{\mu \nu}^{\lambda}$, acts on $g_{\mu \nu}$ and preserves its conformal class $[1,7]$

$$
\triangle_{\omega}^{W} g_{\mu \nu} \equiv \partial_{\omega} g_{\mu \nu}-W_{\omega \mu}^{\lambda} g_{\lambda \nu}-W_{\omega \nu}^{\lambda} g_{\mu \lambda}=2 W_{\omega} g_{\mu \nu}
$$

The Weyl connection can be constructed from the conventional Christoffel connection $\Gamma_{\mu \nu}^{\lambda}$, supplemented by an $W_{\omega}$-dependent expression

$$
W_{\mu \nu}^{\lambda}=\Gamma_{\mu \nu}^{\lambda}+W^{\lambda} g_{\mu \nu}-W_{\mu} \delta_{\nu}^{\lambda}-W_{\nu} \delta_{\mu}^{\lambda}
$$

A curvature tensor is determined as usual by

$$
\left[\triangle_{\mu}^{W}, \triangle_{\nu}^{W}\right] V_{\alpha}=-{ }^{W} R_{\alpha \mu \nu}^{\beta} V_{\beta},
$$

whose traces define "Ricci" quantities

$$
{ }^{W_{\mu \nu}}={ }^{W_{R}}{ }_{\mu \alpha \nu}, \quad{ }^{W} R={ }^{W_{R}}{ }_{\mu} .
$$

The Einstein-Weyl equation then requires that ${ }^{W} R_{(\mu \nu)}$, the symmetric part of the "Ricci" tensor (generically $W_{R_{(\mu \nu)}}$ is not symmetric), be in the same conformal class as the metric tensor,

$$
{ }^{W_{(\mu \nu)}}=\lambda g_{\mu \nu}
$$

or equivalently in three dimensions

$$
{ }^{W_{R}} R_{(\mu \nu)}-\frac{g_{\mu \nu}{ }^{W}}{3} R=0 .
$$

From (23) and $(24){ }^{W} R_{(\mu \nu)}$ can be expressed in terms of the usual Ricci tensor, supplemented by $W_{\omega}$-dependent terms

$$
{ }^{W_{(\mu \nu)}}=R_{\mu \nu}+D_{(\mu} W_{\nu)}+W_{\mu} W_{\nu}+g_{\mu \nu}\left(D_{\lambda} W^{\lambda}-W_{\lambda} W^{\lambda}\right) .
$$

Thus the Einstein-Weyl equation (25) requires the vanishing of a trace free quantity.

$$
R_{\mu \nu}-\frac{1}{3} g_{\mu \nu} R+W_{\mu} W_{\nu}-\frac{g_{\mu \nu}}{3} W^{\lambda} W_{\lambda}+D_{(\mu} W_{\nu)}-\frac{1}{3} g_{\mu \nu} D^{\lambda} W_{\lambda}=0 .
$$

(In (26) and (27) $D_{\omega}$ is the covariant derivative constructed with the Christoffel connection $\Gamma_{\mu \nu}^{\alpha}$.)

The equations (22) and (26) are preserved under conformal transformations: the metric tensor is rescaled and the Weyl potential undergoes a gauge transformation.

$$
g_{\mu \nu} \rightarrow e^{2 \sigma} g_{\mu \nu}, \quad W_{\mu} \rightarrow W_{\mu}+\partial_{\mu} \sigma .
$$


This gauge freedom is fixed by choosing the "Gauduchon" gauge $D^{\mu} W_{\mu}=0$, and one can further show that for positive definite metrics $D_{(\mu} W_{\nu)}$ vanishes

$$
D_{(\mu} W_{\nu)}=0 .
$$

This leaves from (27) the gauge fixed, 3-dimensional Einstein-Weyl equation

$$
R_{\mu \nu}-\frac{1}{3} g_{\mu \nu} R+W_{\mu} W_{\nu}-\frac{g_{\mu \nu}}{3} W^{\lambda} W_{\lambda}=0 .
$$

Comparison with (8) and (9) shows that our 3-dimensional equation for the vanishing of the 4-dimensional Weyl tensor coincide with the gauge-fixed Einstein-Weyl equation, apart from a relative sign between the curvature quantities and the Weyl quantities. This sign discrepancy arises because we performed our reduction on a space with indefinite signature. When the reduction is performed with positive metric, the signs coincide.

This relation to Einstein-Weyl theory puts our work into contact with a wide mathematical literature where solutions other than our (10)-(16) are derived.

\section{Acknowledgements}

This work was supported in part by funds provided by the U.S. Department of Energy under cooperative research agreement \#DF-FC02-94ER40818.

Дуже мені приємно сказати слова на закінчення конференцї, але по Англійскі.

It was very useful and pleasant to participate in this conference. The pleasure came from seeing how active and vibrant physics, mathematics and mathematical physics are in Eastern Europe, specifically Ukraine. The usefulness for me was in the informative comments that I received about my presentation. They will certainly inform my further research.

I am sure that all other participants have similarly benefited from and enjoyed the meeting, which was efficiently run by the local organizers. All of us thank them for their warm Ukrainian hospitality and we look forward to returning in the future to charming Kiev.

\section{References}

[1] Bergmann P.G., Introduction to the theory of relativity, Prentice Hall, New York, 1942.

[2] Grumiller D., Jackiw R., Kaluza-Klein reduction of conformally flat spaces, Intern. J. Modern Phys. D 15 (2006), 2075-2094, math-ph/0609025.

[3] Guralnik G., Iorio A., Jackiw R., Pi S.Y., Dimensionally reduced gravitational Chern-Simons term and its kink, Ann. Physics 308 (2003), 222-236, hep-th/0305117.

[4] Grumiller D., Kummer W., The classical solutions of the dimensionally reduced gravitational Chern-Simons theory, Ann. Physics 308 (2003), 211-221, hep-th/0306036.

[5] Cacciatori S., Calderelli M., Klemm D., Mansi D., More on BPS solutions of $N=2, D=4$ gauged supergravity, JHEP 2004 (2004), no. 7, 061, 31 pages, hep-th/0406238.

Bergamin L., Grumiller D., Iorio A., Nuñez C., Chemistry of Chern-Simons supergravity: reduction to a BPS kink, oxidation to M-theory and thermodynamical aspects, JHEP 2004 (2004), no. 11, 021,40 pages, hep-th/0409273.

Sahoo B., Sen A., BTZ black hole with Chern-Simons and higher derivative terms, JHEP 2006 (2006), no. 7, 008, 11 pages, hep-th/0601228.

Sahoo B., Sen A., $\alpha^{\prime}$-correction to external dyonic black holes in heterofic string theory, JHEP 2007 (2007), no. 1, 010, 18 pages, hep-th/0608182.

[6] Eastwood M., Private communication.

[7] Jones P., Tod K., Minitwistor spaces and Einstein-Weyl spaces, Classical Quantum Gravity 2 (1985), 565577.

Tod K., Compact 3-dimensional Einstein-Weyl structures, J. Lond. Math. Soc. (2) 42 (1992), 341-351. Eastwood M., Tod K., Local constraints on Einstein-Weyl geometries, J. Reine Angew. Math. 491 (1997), $183-198$. 